\title{
PA-117 NEW E-LEARNING TOOL FOR FEMALE GENITAL SCHISTOSOMIASIS: A SUPPLEMENT TO THE WHO POCKET ATLAS OF FGS
}

Solrun Softeland, ${ }^{1}$ Motshedisi Sebitloane, ${ }^{2}$ Bellington Vwalika, ${ }^{3}$ Myra Taylor, ${ }^{2}$ Hashini Galappaththi-Arachchige, ${ }^{1}$ Sigve Holmen, ${ }^{1}$ Svein Gunnar Gundersen, ${ }^{5}$ Patricia Ndhlovu, ${ }^{5}$ Eyrun Floerecke Kjetland'. ' Oslo University Hospital, Norway; ${ }^{2}$ UKZN, South Africa; ${ }^{3}$ UTH, Lusaka, Zambia; ${ }^{4}$ University of Adger, Norway; Imperial College London, United Kingdom

10.1136/bmjgh-2016-000260.146

Background Schistosomiasis affects 261 million people worldwide, most of them in Africa. Female genital schistosomiasis (FGS) may cause abnormal vaginal discharge, contact bleeding, genital tumours, infertility, ectopic pregnancies and increased susceptibility to HIV. Visualisation of lesions is the key to diagnosis but there is little knowledge about FGS among health professionals. In order to facilitate the use of the WHO pocket atlas for FSG, we present an elearning module for medical students in endemic areas. The e-learning material is usable on smartphones, and in areas with low internet speed.

Methods Two FGS atlases form the platform for the elearning: The First Colposcopic Atlas of Schistosomiasis in the Lower Female Genital Tract (Norseth et al. 2014) and The WHO Pocket Atlas for FGS (WHO, 2015). Actors were recruited for demonstration of the examination techniques. Medical students were approached to explore their current elearning platforms. Website creators of two existing elearning modules were invited to collaborate. The project is part of a larger project that was granted permissions by the Biomedical Research Ethics Administration, University of KwaZulu-Natal (KZN), South Africa.

Results A new e-learning tool is presented: all lesions, history taking and the examination techniques for identification of FGS are shown. There is a post-learning quiz for selfevaluation. Medical students in an endemic area were asked to give a qualitative evaluation on the learning outcome.

Conclusions There is a need to raise the index of suspicion for FGS as a differential diagnosis among health care professionals. This e-learning may contribute to the dissemination of knowledge of FGS to all health care professionals who can access the internet when furthering knowledge in clinical practice. Furthermore, there is a need to disseminate knowledge to professionals who may not be using the internet. 